\title{
Creutzfeldt-Jakob disease with congophilic kuru plaques: CT and pathological findings of the cerebral white matter
}

\author{
Akihiro Kawata, Masakazu Suga, Masaya Oda, Hideaki Hayashi, Hitoshi Tanabe
}

\begin{abstract}
In a patient whose Creutzfeldt-Jakob disease with congophilic kuru plaques that was proved at necropsy, the early brain CT showed low-density areas in the cerebral white matter before cortical atrophy and ventricular enlargement became apparent. Subsequently, there occurred diffuse white matter lucency and severe brain atrophy. At necropsy, there was severe white matter destruction which was more prominent than cortical neuronal loss. Serial CT scans were of great value for demonstrating the early and predominant changes in the cerebral white matter.
\end{abstract}

$(\mathcal{F}$ Neurol Neurosurg Psychiatry 1992;55:849-851)

Congophilic kuru plaques rarely occur in the brain in sporadic cases of Creutzfeldt-Jakob disease (CJD). ${ }^{1}$ The disease CJD with congophilic kuru plaques (CJD-KP), is clinically characterised by an unusually long duration of illness for CJD and ataxia usually preceding dementia, but there is considerable clinical variability.' Neuropathological features other than kuru plaques include variable degrees of spongiform changes in the cerebral cortex and frequent involvement of the cerebral white matter. $^{2}$ Despite the unique clinicopathological features, there has been very little information about the neuroradiological findings. We report here a necropsy case of CJD$\mathrm{KP}$, with special reference to the cerebral white-matter changes revealed by CT.

\section{Case report}

Tokyo Metropolitan Neurological Hospital, 2-6-1, Musashidai, Fuchu-shi, Tokyo 183 Japan

Department of

Neurology

A Kawata

M Suga

H Hayashi

H Tanabe

Department of

Neuropathology

M Oda

Correspondence to:

Dr A Kawata

Received 28 August 1991

and in revised form

12 December 1991.

Accepted 12 December

1991 pleocytosis. Five months after admission, she was confined to bed and her condition rapidly deteriorated. In early September, she was in the state of akinetic mutism and showed muscle rigidity, areflexia in the four limbs, myoclonus in the face and four limbs, and a startled response to sounds. An EEG showed a background activity of $3-5 \mathrm{~Hz}$ with intermittent synchronous $100 \mu \mathrm{V} \theta$ activity in the fronto-centro-parietal region. The clinical pictures remained relatively unmodified over the next three years; the intensity of myoclonus progressively decreased; the intermittent discharges of the EEG gradually disappeared to be replaced by a slow monomorphous activity by July 1985 . On 8 December 1987, she died of respiratory failure. The duration of the disease was 41 months.

The initial CT scan performed in February of 1984 revealed a bilateral pallidal calcification and low attenuation in the cerebral white matter without cortical atrophy and ventricular dilatation (fig 1A, B). The low density of the white matter was accentuated at the cerebral subcortex. A second CT scan obtained six months after the initial CT scan, indicated moderate enlargement of the ventricles with diffuse white-matter lucency (leuko-araiosis). A dramatic enlargement of the ventricles and widening of the cerebral and cerebellar sulci developed within four months after the state of akinetic mutism appeared (fig 1C, D).

At necropsy, focal pneumonia and mild arteriosclerosis were found. The brain weighed 805 grams and was markedly atrophic with cystic softening of the white matter at the cerebral subcortex (fig 2A). There was marked atrophy of the cerebellum and brainstem. Microscopically, there was severe spongy degeneration and less marked diffuse neuronal loss of the cerebral cortex, thalamus and basal ganglia. Diffuse loss of myelinated nerve fibres accompanied with proliferation of hypertrophic astrocytes and fat-laden macrophages were seen throughout the cerebral white matter. At the subcortical white matter of the cerebrum, there were circumscribed necrotic foci. In the cerebellum, both Purkinje and granule cells were diminished. Numerous congophilic kuru like plaques were present in the cerebellar cortex (fig 2B), and to a lesser extent in the cerebral cortex.

Experimental transmission of the disease to mice through inoculation of the brain homogenates was successful. To distinguish the present case from Gerstmann-Sträussler syn- 

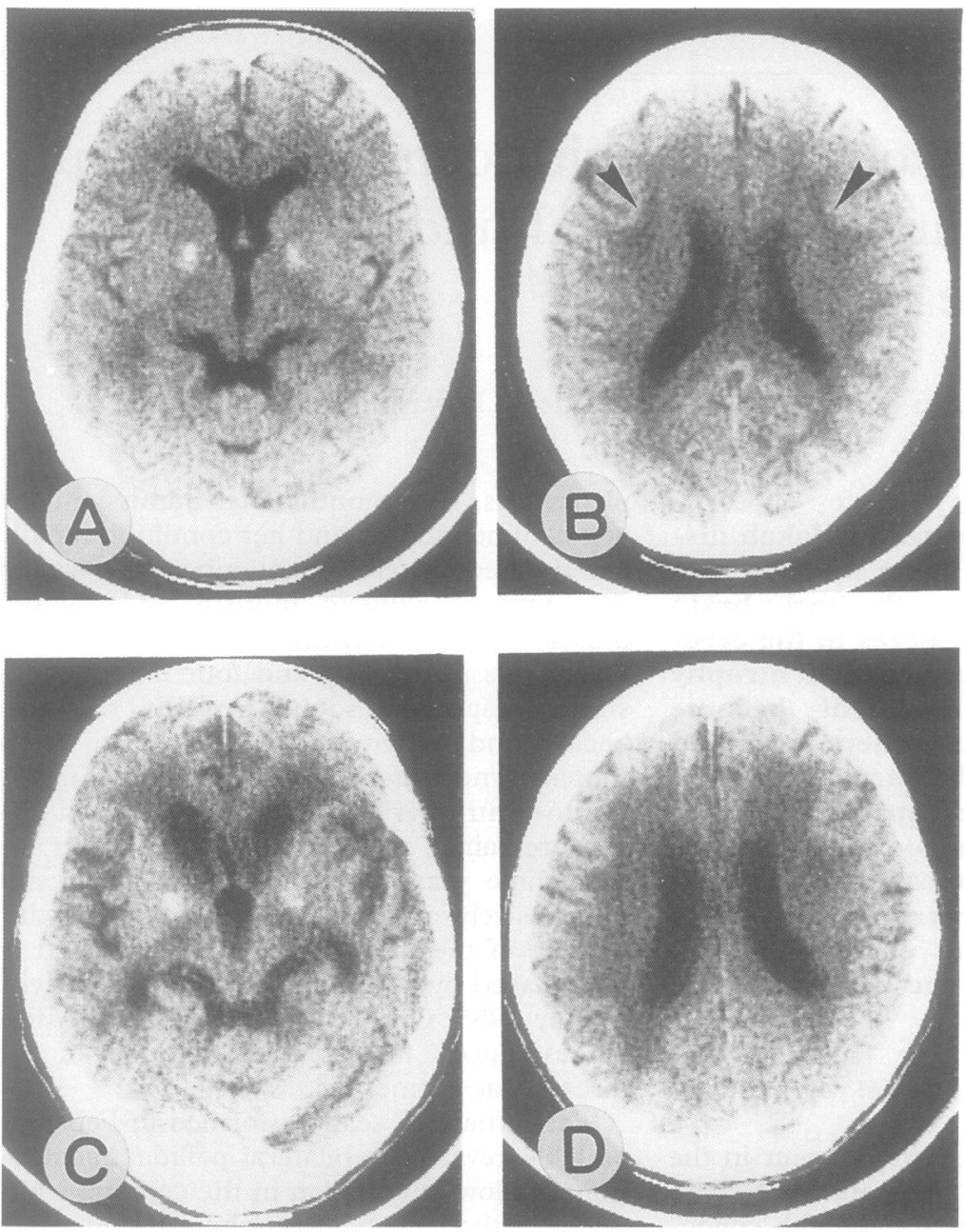

Figure 1 A, B) CT scans (February 1984) show a bilateral pallidal calcification and low attenuation in the cerebral white matter without cortical atrophy and ventricular dilatation. The low-density is accentuated at the cerebral subcortex (arrow heads). $C, D) C T$ (fanuary 1985) shows remarkable enlargement of the lateral ventricles with diffuse white matter lucency. nine-to-valine change at codon $129^{4}$ was carried heterozygously.

\section{Discussion}

The patient showed clinical features of CJD, but the duration of the illness was longer than that of typical CJD. Neuropathological, molecular genetic and transmission studies confirmed the diagnosis of CJD-KP.

The most prominent CT finding in our patient was the appearance of low-density areas in the cerebral white matter before cortical and central atrophy became apparent. Subsequent enlargement of ventricles progressed more rapidly than the cerebral cortical atrophy, suggesting that the involvement of white matter was more severe than the involvement of the cortex. With regard to the CT finding, previous reports of CJD-KP mention only cortical atrophy and ventricular dilatation of the brain, but no white-matter changes have been described. ${ }^{56}$ On the other hand, the CT imagings of CJD without kuru plaques show bilateral cortical atrophy and no apparent white-matter changes. ${ }^{78}$ The white-matter abnormalities, if present, develop during the final stage. ${ }^{8}$ Recent study with MRI also showed increased signal intensity in the basal ganglia, thalamus and cerebral cortex without significant change in the white matter. ${ }^{910}$ The discrepancy in the occurrence of white-matter changes suggests the wide spectrum of pathology in CJD patients.

The neuropathological findings of this patient closely matched the CT images, showing diffuse marked degeneration with necrotic foci in the cerebral white matter. Such whitematter lesions seem to be an exceptional finding in CJD, because the main affected area in CJD has been regarded as cortical and subcortical grey matter. However, extensive degeneration of cerebral white matter which cannot be explained simply as secondary to cortical degeneration has been described in the patients with CJD-KP and a special type of CJD without KP. ${ }^{1112}$ The outstanding whitematter lesions observed in this patient are not only due to the long clinical course, since the
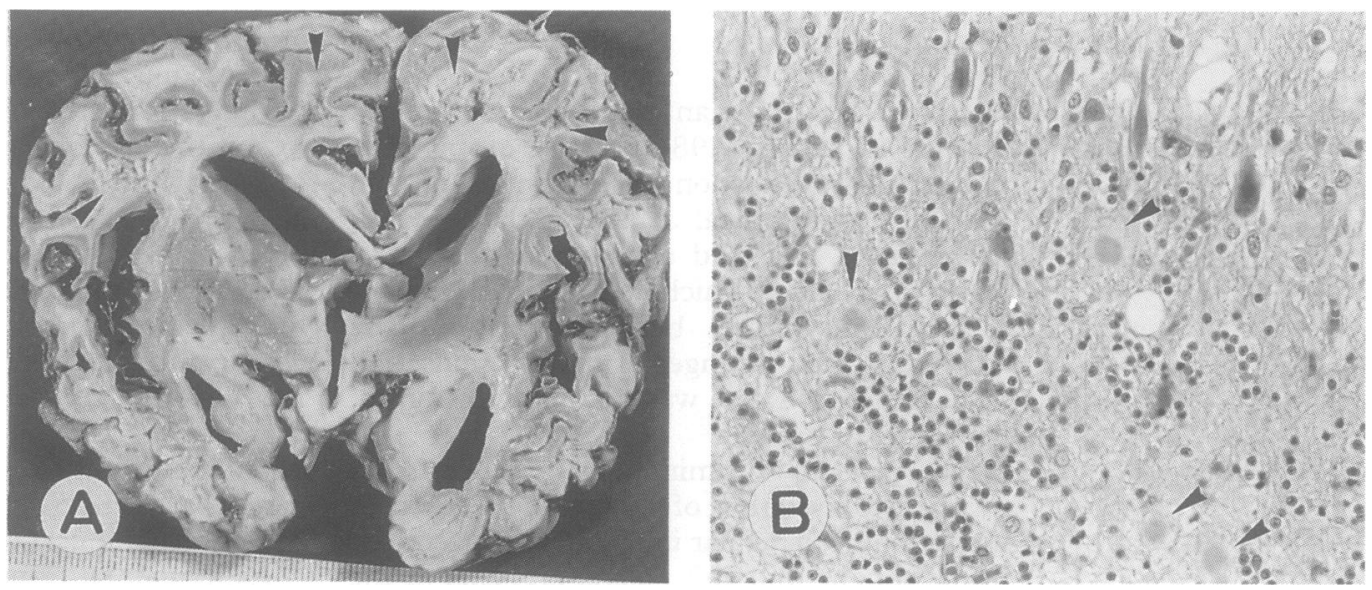

Figure 2 A) Coronal section of the cerebral hemispheres showing overall necrosis with cystic softening (arrow heads) of the white matter. B) Photomicrograph of the cerebellar cortex showing kurulike amyloid plaques (arrow heads). H-E staining; $206 \times$ 
initial CT scan which was carried out before the onset of akinetic mutism had already revealed white-matter changes without cortical atrophy. They are probably related to the difference in the strain of virus and/or the susceptibility to the virus influenced by the host genotype.

In conclusion, we speculate that the whitematter changes revealed by CT in this patient probably reflected the disease process primarily affecting the cerebral white matter. Whether the white-matter changes reported here are general findings of CJD-KP without known GSS-associated PrP variants remains to be determined. However, our results indicate that CT scans or the more sensitive MRI might reveal the white-matter changes characteristic for some types of CJD and may be helpful in detecting the disease process before death.

We are grateful to Drs J Tateishi, and K Doh-ura, Department of Neuropathology, Neurological Institute, Kyushu University Medical School, for performing the transmission and molecular genetic studies.

1 Masters CL, Gajdusek DC, Gibbs CJ Jr. Creutzfeldt-Jakob disease virus isolations from the Gerstmann-Sträussler syndrome with an analysis of the various forms of amyloid plaque deposition in the virus-induced spongiform encephalopathies. Brain 1981;104:559-88.

2 Yagishita S. Creutzfeldt-Jakob disease with kuru-like plaques in Japan. Acta Pathol fpn 1981;31(6):923-42.

3 Hsiao K, Prusiner SB. Inherited human prion diseases. Neurology 1990;40:1820-7.

4 Owen F, Poulter M, Collinge J, Crow TJ. Codon 129 changes in the prion protein gene in Caucasians. $\mathrm{Am} \mathcal{F}$ changes in the prion protein
Hum Genet 1990;46:1215-16.

5 Pearlman RL, Towfighi J, Pezeshkpour GH, Tenser RB, Turel AP. Clinical significance of types of cerebellar amyloid plaques in human spongiform encephalopathies. amyloid plaques in human spon

6 Okuda S, Indo T, Goto H, Hashizume Y. An autopsy case of Creutzfeldt-Jakob disease with white matter lesion and amyloid plaques. Clin Neurol 1986;26:261-9.

7 Rao CVGK, Brennan TG, Garcia JH. Computed tomography in the diagnosis of Creutzfeldt-Jakob disease. $f$ Comput Assist Tomogr 1977;1:211-15.

8 Kovanen J, Erkinjuntti T, Iivanainen M, Ketonen L, Haltia M, Sulkava R, Sipponen JT. Cerebral MR and CT imaging in Creutzfeldt-Jakob disease. $f$ Comput Assist Tomogr 1985;9:125-8.

9 Gertz H-J, Henkes H, Cervos-Navarro J. Creutzfeldt-Jakob disease: correlation of MRI and neuropathologic findings. Neurology 1988;38:1481-2.

10 Milton WI, Atlas SW, Lavi E, Mollman JE. Magnetic resonance imaging of Creutzfeldt-Jakob disease. Ann resonance imaging of
Neurol 1991;29:438-40.

11 Mizutani T, Okumura A, Oda M, Shiraki H. Panencephalopathic type of Creutzfeldt-Jakob disease: primary involvement of the cerebral white matter. $\mathcal{F}$ Neurol Neurosurg Psychiatry 1981;44:103-15.

12 Macchi G, Abbamondi AL, Di Trapani G, Sbriccoli A. On the white matter lesions of the Creutzfeldt-Jakob disease. Can a new subentity be recognized in man? $\mathcal{F}$ Neurol Sci 1984;63:197-206. 